\title{
HEALTH STATUS OF DATE MUSSEL "LITHOPHAGA LITHOPHAGA, FAMILY: MYTILIDAE" IN ALEXANDERIA, EGYPT: POTENTIAL EFFECTS OF THE HEAVY METAL POLLUTION
}

\author{
Wesam M. Salama ${ }^{1 *}$; Mohamed H. Mona ${ }^{1}$; Aml Z. Ghoneium ${ }^{2}$ \\ ${ }^{1}$ Zoology Department, Faculty of Science, Tanta University, Gharbia, Egypt \\ ${ }^{2}$ Zoology Department, Faculty of Science, Damanhour University, Beheira, Egypt
}

\begin{abstract}
Article History:
Received: 29 March 2020

Revised: 9 April 2020

Accepted: 10 April 2020

Published Online:

22 April 2020

Keywords:

Bivalve molluscs

Heavy metals

Lithophaga lithophaga

Pathology

Seawater pollution

*Correspondence:

Wesam Salama

Zoology Department

Faculty of Science

Tanta University

Gharbia, Egypt

E-mail:

wesam.hassan@science. tanta.edu.eg

ABSTRACT

The date mussels (Lithophaga lithophaga) are rock-boring bivalve molluscs that are generally used as bio-indicators for pollution. They can accumulate pollutants in their tissues, due to their filter feeding behaviour. Therefore, the current study aimed to use the date mussels to evaluate the heavy metal pollution in three coastal areas of Alexandria (Abo Qir, Alasafra, and Miami) during the four seasons to determine the most polluted area. The current study also aimed to evaluate the health status and disease burden of the date mussels collected from the most polluted area according to the World Organisation for Animal Health (OIE) manual. The results indicated that $\mathrm{Pb}, \mathrm{Cd}$, and $\mathrm{Cu}$ increased significantly in seawater of Abo Qir area during summer, and was correlated with their accumulation in the mussel tissues. As a consequence of pollution, a significant decrease in the amount of dissolved oxygen was observed in the seawater of Abo Qir area during summer. The pathological examination of samples from Abo Qir area using OIE manual showed that the mussels had signs of weakness from the outer shell examination as gaping, fragility, discolouration, and fouling. Moreover, infection with ciliates was observed in the date mussel tissues collected from the most polluted area. Interestingly, abnormal case of disseminated neoplasia or sarcoma also appeared under the effect of pollution. In conclusion, Abo Qir is the most polluted area with low dissolved oxygen concentration during summer. Moreover, accumulation of heavy metals in the soft parts of the date mussels could increase their disease susceptibility.
\end{abstract}

\section{INTRODUCTION}

Date mussels (Lithophaga lithophaga Linnaeus, 1758) are bivalved Mollusca that considered one of the boring mussels on any calcareous substrate ${ }^{[1]}$. Environmental variations and marine pollution may affect date mussels in Egypt. Heavy metals may enter aquatic ecosystem from different sources as natural and anthropogenic sources, which include industrial or domestic sewage ${ }^{[2]}$. Mussels are recognized as pollution bio-indicator organisms because 
they accumulate pollutants in their soft tissues due to their filter feeding habits ${ }^{[3]}$. Interestingly, bivalved Mollusca vulnerable to be infected with many pathogens as viruses, fungi, bacteria, protozoa due to heavy metals pollution that can induce immune-suppression and reduction in disease resistance ${ }^{[4]}$. The interactions between the two kinds of stresses as infectious diseases and pollutants on aquatic organisms may cause synergistic effect that cause severe damage to a Molluscan population $^{[5]}$. Protozoal infection considered the major threats for farmed and natural bivalved species $^{[6]}$. It is well known that heavy metals bioaccumulation and thermal pollution have a major role in viral disease outbreaks in marine invertebrates ${ }^{[7]}$. Another abnormal condition was noticed in Mollusca exposed to pollution and infectious disease such as disseminated neoplasia or sarcomas; it defined as a proliferation of abnormal circulating cells of unknown origin and defined as cancerous condition of bivalvia ${ }^{[8]}$. The current study aimed to evaluate the heavy metals levels in seawater and their bioaccumulation in soft parts of boring date mussels that collected from different coastal areas in Alexandria during the four seasons, as a bio-indicator for aquatic pollutant, and investigated the health status of date mussels under the effect of pollution.

\section{MATERIAL AND METHODS Studying area and sampling:}

Three locations were chosen from the coastal regions of Alexandria (Abu Qir, Alasafra, and Miami, Figure 1) to study the pollution with heavy metals during the four seasons in 2018. Water samples were seasonally collected in the morning (5 replica/location/season) at depth of 3-5 m, filtered to remove any debris, and stored at $-20^{\circ} \mathrm{C}$ until use. Molluscan samples "Lithophaga lithophaga" were collected seasonally from the three collection sites, as pollution bio-indicators; whereas, samples were collected from the most polluted area during the significant season to evaluate of the disease burden of date mussels under the effect of pollution. Samples were collected by scuba divers who break the rocks with special sledge hammers without detrimental effect on the organisms living on their surfaces and within substratum. Collected samples ( 50 samples/location/season) were transferred within two hours in ice-box to central laboratory in Tanta University, divided into groups, and labelled. Diameters or morphometric analysis of samples were measured; and samples were dissected for heavy metals analysis, biochemical compositions, and disease detection using World Organisation for Animal Health (OIE) manual.

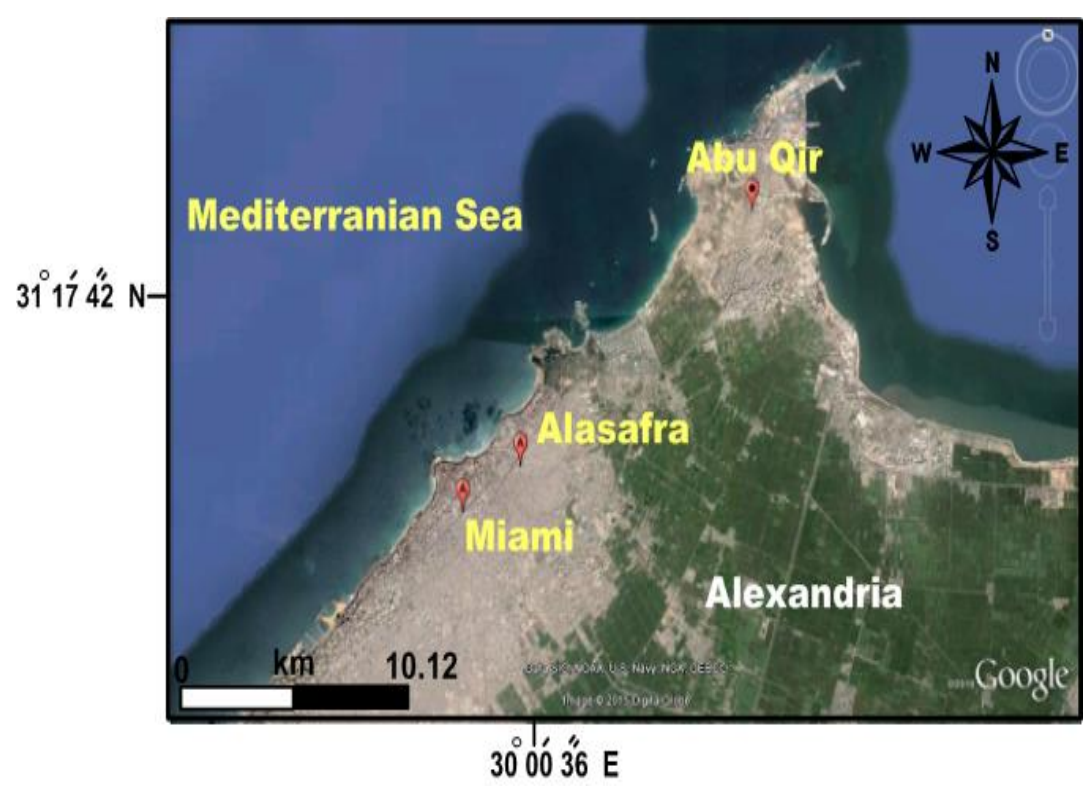

Figure 1: Google maps showing the collection sites along Alexandria coastal line. 


\section{Physicochemical properties of water samples}

Surface water samples were collected five times from coastal regions of Alexandria during the four seasons for measuring of $\mathrm{pH}$, dissolved oxygen, temperature, and salinity. The value of $\mathrm{pH}$ was measured immediately after collection using Bench type $\mathrm{pH}$ meter (3510 Electrochemistry Analyser $\mathrm{pH}$ meter; Jenway, Stone, UK). Dissolved oxygen was determined according to the method of Grasshoff $^{[9]}$. Temperature was measured using an ordinary thermometer. Salinity was determined by measuring the electrical conductivity using an inductive salinometer (model RS.10; Beckman, Cedar Grove, NJ, USA).

\section{Heavy metal analysis}

The heavy metals in both water and tissue samples were analysed according to the method of Bernhard ${ }^{[10]}$. The heavy metals of seawater samples were determined using Graphite Furnace Atomic Absorption Spectroscopy (model 2380; Perkin-Elmer, Shelton, CT, USA). Soft parts from dissected samples were digested in Teflon vessels using concentrated $\mathrm{HNCO}_{3}$, dissolved in deionized distilled water, and analysed using ion selective electrode (AVL, 9180 electrolyte analyser, Roswell, GA, USA).

\section{Pathological techniques listed by OIE}

Date mussels "50 samples" were collected from Abo Qir area (the most polluted area) in summer and examined according to $\mathrm{OIE}^{[11]}$ to detect symptoms, clinical aspects, and pathological conditions using macroscopic, microscopic (histological), and ultrastructural examinations. In macroscopic or gross examination, mussels were examined for any external clinical abnormalities and clinical alterations. Parts of gills, mantle, gonads, and digestive tract were removed from the dissected samples and fixed in $10 \%$ neutral buffered formalin (for histopathological examination), or fixed in $2.5 \%$ glutaraldehyde solution and osmium tetroxide (for transmission electron microscope, TEM). The fixed tissues with formalin were dehydrated and embedded in paraffin wax. Then, thin sections were prepared, mounted, and stained using haematoxylin and eosin $(\mathrm{H} \& \mathrm{E})$ or toluidine blue. For TEM, the fixed tissues were washed with phosphate buffer and dehydrated with ascending grades of ethanol, and embedded in Epon 812 (Sigma-Aldrich Chemie GmbH, Taufkirchen, Germany).

\section{Statistical analysis}

All data are the means of five replicates. Normality of the data was tested with the Kolmogorov-Smirnov test. Two-way analysis of variance (ANOVA) was used to test the effects of sites (3 levels) and seasons (4 levels) on the properties of seawater sample and the heavy meats concentrations of seawater samples and soft tissues of date mussels' samples. If there is a significant difference between means, Tukey's post-hoc comparisons among different groups were performed. $P$-values $<0.05$ were considered to be statistically significant.

\section{RESULTS}

\section{Physicochemical parameters of seawater samples}

Measurements of some physicochemical parameters of seawater samples from the three studying area during the four seasons were shown in Table "1". The results indicated that there are a significant increase $(P<0.05)$ in $\mathrm{pH}$ of seawater samples collected from Abo Qir in summer and a significant decrease in $\mathrm{pH}$ of seawater samples collected from Alasafra and Miami in winter. In addition, a significant decrease $(P<0.05)$ in the amount of dissolved oxygen was recorded in seawater samples collected from Abo Qir in almost all seasons, while it increased significantly in seawater samples collected from Alasafra and Miami in almost all seasons (4.0-4.6 mg/L, versus 5.7-5.9 and 5.0-5.7 mg/L, respectively). 
Table 1: Physicochemical properties of seawater samples from the studying area during the four seasons.

\begin{tabular}{|c|c|c|c|c|c|c|c|c|c|c|c|c|}
\hline & \multicolumn{4}{|c|}{ Abo-Qir } & \multicolumn{4}{|c|}{ Al-Asafra } & \multicolumn{4}{|c|}{ Miami } \\
\hline & Sum & Aut & Win & Spr & Sum & Aut & Win & Spr & Sum & Aut & Win & Spr \\
\hline $\mathrm{pH}$ & $\begin{array}{c}8.4 \\
\pm 1.3 \\
\mathrm{a}\end{array}$ & $\begin{array}{c}7.5 \\
\pm 0.3 \\
a b\end{array}$ & $\begin{array}{c}7.6 \\
\pm 1.0 \\
a b\end{array}$ & $\begin{array}{c}8.0 \\
\pm 1.2 \\
\text { ab }\end{array}$ & $\begin{array}{c}8.1 \\
\pm 1.0 \\
\mathrm{ab}\end{array}$ & $\begin{array}{c}7.5 \\
\pm 1.1 \\
a b\end{array}$ & $\begin{array}{c}6.8 \\
\pm 0.4 \\
\mathrm{~b}\end{array}$ & $\begin{array}{c}8.0 \\
\pm 1.7 \\
a b\end{array}$ & $\begin{array}{c}8.0 \\
\pm 2.0 \\
a b\end{array}$ & $\begin{array}{c}7.4 \\
\pm 1.6 \\
a b\end{array}$ & $\begin{array}{c}7.2 \\
\pm 0.02 \\
\mathrm{~b}\end{array}$ & $\begin{array}{c}8.0 \\
\pm 1.3 \\
\text { ab }\end{array}$ \\
\hline $\begin{array}{c}\text { Dissolved } \\
\text { oxygen } \\
(\mathrm{mg} / \mathrm{L})\end{array}$ & $\begin{array}{c}4.0 \\
\pm 0.2 \\
\mathrm{~d}\end{array}$ & $\begin{array}{c}4.3 \\
\pm 0.5 \\
\mathrm{~d}\end{array}$ & $\begin{array}{c}4.5 \\
\pm 1.0 \\
\mathrm{~d}\end{array}$ & $\begin{array}{c}4.6 \\
\pm 0.6 \\
\text { cd }\end{array}$ & $\begin{array}{c}5.7 \\
\pm 0.04 \\
\mathrm{ab}\end{array}$ & $\begin{array}{c}5.8 \\
\pm 1.1 \\
\mathrm{ab}\end{array}$ & $\begin{array}{c}5.9 \\
\pm 0.8 \\
\mathrm{a}\end{array}$ & $\begin{array}{c}5.9 \\
\pm 1.0 \\
\mathrm{a}\end{array}$ & $\begin{array}{c}5.6 \\
\pm 0.7 \\
a b\end{array}$ & $\begin{array}{c}5.7 \\
\pm 0.1 \\
a b\end{array}$ & $\begin{array}{c}5.1 \\
\pm 1.3 \\
\mathrm{ab}\end{array}$ & $\begin{array}{c}5.0 \\
\pm 1.1 \\
\mathrm{bc}\end{array}$ \\
\hline $\begin{array}{c}\text { Temperature } \\
\left({ }^{\circ} \mathrm{C}\right)\end{array}$ & $\begin{array}{c}30.3 \\
\pm 2.0 \\
\mathrm{a}\end{array}$ & $\begin{array}{l}28.5 \\
\pm 3.0 \\
c\end{array}$ & $\begin{array}{c}28.5 \\
\pm 1.7 \\
\mathrm{bc}\end{array}$ & $\begin{array}{c}28.0 \\
\pm 1.6 \\
\mathrm{c}\end{array}$ & $\begin{array}{c}29.8 \\
\pm 0.04 \\
\mathrm{ab}\end{array}$ & $\begin{array}{c}28.3 \\
\pm 2.0 \\
\mathrm{bc}\end{array}$ & $\begin{array}{c}28.2 \\
\pm 1.8 \\
\mathrm{c}\end{array}$ & $\begin{array}{l}28.0 \\
\pm 2.5 \\
\mathrm{c}\end{array}$ & $\begin{array}{c}30.2 \\
\pm 3.2 \\
\mathrm{a}\end{array}$ & $\begin{array}{c}28.0 \\
\pm 1.1 \\
\mathrm{c}\end{array}$ & $\begin{array}{c}27.5 \\
\pm 2.0 \\
c\end{array}$ & $\begin{array}{l}27.7 \\
\pm 1.8 \\
c\end{array}$ \\
\hline $\begin{array}{c}\text { Salinity } \\
\text { (ppt) }\end{array}$ & $\begin{array}{l}35.7 \\
\pm 1.6 \\
\mathrm{a}\end{array}$ & $\begin{array}{r}35.3 \\
\pm 1.0 \\
\mathrm{a}\end{array}$ & $\begin{array}{c}35.4 \\
\pm 2.3 \\
\mathrm{a}\end{array}$ & $\begin{array}{l}35.1 \\
\pm 1.4 \\
\mathrm{a}\end{array}$ & $\begin{array}{l}35.0 \\
\pm 1.0 \\
\mathrm{a}\end{array}$ & $\begin{array}{l}34.5 \\
\pm 0.8 \\
\mathrm{a}\end{array}$ & $\begin{array}{c}34.6 \\
\pm 1.4 \\
\mathrm{a}\end{array}$ & $\begin{array}{c}34.6 \\
\pm 2.0 \\
\mathrm{a}\end{array}$ & $\begin{array}{r}35.0 \\
\pm 0.6 \\
\mathrm{a}\end{array}$ & $\begin{array}{l}34.5 \\
\pm 2.0 \\
\mathrm{a}\end{array}$ & $\begin{array}{l}34.0 \\
\pm 2.4 \\
\mathrm{a}\end{array}$ & $\begin{array}{c}34.5 \\
\pm 2.4 \\
\mathrm{a}\end{array}$ \\
\hline
\end{tabular}

Data are presented as means \pm standard deviation $(n=5)$. Sum: summer, Aut: autumn, Win: winter, Spr: spring, ppt: parts per thousand. Values with different letters in the same row were significantly different $(P<0.05)$.

Seawater temperature was ranged between 27.5 to $30.3^{\circ} \mathrm{C}$ during the four seasons in all stations. There are no significant differences in salinity of seawater in all locations during the four seasons (Table 1).

Measurements of heavy metals in the seawater samples from the three studying areas indicated that the highest concentration of most heavy metals was found in seawater samples of Abo Qir during summer (Table 2). Obviously three of the eight evaluated heavy metals $(\mathrm{Pb}, \mathrm{Cd}$, and $\mathrm{Cu})$ were significantly increased $(P<0.05)$ in shallow seawater collected from Abo Qir during summer compared with Alasafra and Miami shallow seawater at the same season.

\section{Morphometric analysis and heavy metal concentrations of date mussels}

There were no differences in shell heights, total length, and width of date mussels collected from the three locations (data not shown). While, samples collected from Alasafra and Miami in summer showed a slight increase, but insignificant, in the total weights compared with samples collected from Abo Qir (data not shown). On the other hand, significant increases $(P<0.05)$ in the concentrations of " $\mathrm{Pb}, \mathrm{Cd}$, and $\mathrm{Cu}$ " were recorded in the samples collected from Abo Qir compared with the other sites during summer (Table 3). The relation between values of heavy metals in both seawater and soft parts of date mussels changed significantly in most cases according to the collection sites and seasons (Table 4). Different collection sites and season could significantly $(P<0.05)$ affect concentrations of $\mathrm{Fe}, \mathrm{Ni}, \mathrm{Pb}$, and $\mathrm{Co}$ in seawater, and $\mathrm{Ni}, \mathrm{Pb}, \mathrm{Cd}, \mathrm{Cu}, \mathrm{Co}$, and $\mathrm{Cr}$ in soft parts of date mussels.

\section{Pathological examination of Abo Qir date mussels according to OIE}

Macroscopic examination of 50 samples of Abo Qir date mussels collected during summer showed that 33 samples $(66 \%)$ had external abnormal symptoms and signs of weakness such as fouling, shell discolouration, shell fragility (Figure 2) and 
Table 2: Heavy metals $(\mathrm{g} / \mathrm{L})$ in the seawater samples from the studying area during the four seasons.

\begin{tabular}{|c|c|c|c|c|c|c|c|c|c|c|c|c|}
\hline & \multicolumn{4}{|c|}{ Abo-Qir } & \multicolumn{4}{|c|}{ Alasafra } & \multicolumn{4}{|c|}{ Miami } \\
\hline & Sum & Aut & Win & Spr & Sum & Aut & Win & Spr & Sum & Aut & Win & Spr \\
\hline $\mathrm{Fe}$ & $\begin{array}{c}6.5 \\
\pm 3.4 \\
a\end{array}$ & $\begin{array}{c}6.3 \\
\pm 3.2 \\
a b\end{array}$ & $\begin{array}{c}5.0 \\
\pm 2.3 \\
\mathrm{bc}\end{array}$ & $\begin{array}{c}5.5 \\
\pm 2.5 \\
\mathrm{bc}\end{array}$ & $\begin{array}{c}5.9 \\
\pm 2.0 \\
a b\end{array}$ & $\begin{array}{c}5.6 \\
\pm 1.7 \\
\mathrm{ab}\end{array}$ & $\begin{array}{r}J \\
\pm 1 \\
\text { al }\end{array}$ & & & $\begin{array}{c}3.9 \\
\pm 0.8 \\
\text { cd }\end{array}$ & \pm 0.6 & $\begin{array}{c}3.8 \\
\pm 0.7 \\
d\end{array}$ \\
\hline $\mathrm{Ni}$ & $\begin{array}{c}2.1 \\
\pm 1.0 \\
a b\end{array}$ & $\begin{array}{c}1.9 \\
\pm 0.8 \\
\mathrm{a}\end{array}$ & $\begin{array}{c}1.5 \\
\pm 0.6 \\
\text { abcd }\end{array}$ & $\begin{array}{c}1.7 \\
\pm 0.7 \\
\text { abcd }\end{array}$ & $\begin{array}{c}1.3 \\
\pm 0.4 \\
\text { abcd }\end{array}$ & $\begin{array}{c}1.1 \\
\pm 0.2 \\
\text { bcd }\end{array}$ & $\begin{array}{c}0.8 \\
\pm 0 . \\
\text { d }\end{array}$ & $\begin{array}{c}0.9 \\
\pm 0.2 \\
\mathrm{~d}\end{array}$ & & $\begin{array}{c}1.1 \\
\pm 0.1 \\
\text { bcd }\end{array}$ & $\begin{array}{c}1.0 \\
\pm 0.1 \\
\text { bcd }\end{array}$ & $\begin{array}{c}0.9 \\
\pm 0.1 \\
\mathrm{~cd}\end{array}$ \\
\hline $\mathrm{Pb}$ & $\begin{array}{c}2.4 \\
\pm 1.5 \\
\mathrm{a}\end{array}$ & $\begin{array}{c}2.3 \\
\pm 1.2 \\
\mathrm{a}\end{array}$ & $\begin{array}{c}2.0 \\
\pm 0.9 \\
\mathrm{a}\end{array}$ & $\begin{array}{c}2.1 \\
\pm 1.0 \\
\mathrm{a}\end{array}$ & $\begin{array}{c}1.0 \\
\pm 0.6 \\
\mathrm{~b}\end{array}$ & $\begin{array}{c}0.9 \\
\pm 0.5 \\
\mathrm{~b}\end{array}$ & $\begin{array}{c}0.6 \\
\pm 0.4 \\
\mathrm{~b}\end{array}$ & b & $\begin{array}{c}0.8 \\
\pm 0.2 \\
\mathrm{~b}\end{array}$ & $\begin{array}{c}0.6 \\
\pm 0.1 \\
\mathrm{~b}\end{array}$ & $\begin{array}{c}0.6 \\
\pm 0.1 \\
\mathrm{~b}\end{array}$ & $\begin{array}{c}0.7 \\
\pm 0.1 \\
\mathrm{~b}\end{array}$ \\
\hline $\mathrm{Zn}$ & $\begin{array}{c}5.8 \\
\pm 2.1 \\
a b\end{array}$ & $\begin{array}{c}5.5 \\
\pm 2.0 \\
a b\end{array}$ & $\begin{array}{c}5.2 \\
\pm 1.8 \\
\mathrm{~b}\end{array}$ & $\begin{array}{c}4.9 \\
\pm 1.5 \\
\mathrm{~b}\end{array}$ & $\begin{array}{c}6.0 \\
\pm 0.1 \\
a b\end{array}$ & $\begin{array}{c}5.7 \\
\pm 0.1 \\
a b\end{array}$ & $\begin{array}{c}5.4 \\
\pm 0.04 \\
a b\end{array}$ & $\begin{array}{c}5.3 \\
\pm 0.02 \\
\text { ab }\end{array}$ & $\begin{array}{c}6.3 \\
\pm 1.0 \\
\mathrm{a}\end{array}$ & $\begin{array}{c}5.5 \\
\pm 0.8 \\
\mathrm{ab}\end{array}$ & $\begin{array}{c}5.2 \\
\pm 0.6 \\
a b\end{array}$ & $\begin{array}{c}5.0 \\
\pm 0.5 \\
a b\end{array}$ \\
\hline $\mathrm{Cd}$ & $\begin{array}{c}2.8 \\
\pm 1.4 \\
\mathrm{a}\end{array}$ & $\begin{array}{c}2.6 \\
\pm 1.2 \\
\mathrm{a}\end{array}$ & $\begin{array}{c}2.1 \\
\pm 0.9 \\
a\end{array}$ & $\begin{array}{c}2.4 \\
\pm 1.0 \\
\mathrm{a}\end{array}$ & $\underset{\mathrm{b}}{ \pm 0.4}$ & $\begin{array}{c}0.9 \\
\pm 0.2 \\
\mathrm{bc}\end{array}$ & $\begin{array}{c} \pm 0.2 \\
\mathrm{bc}\end{array}$ & $\begin{array}{c}0.7 \\
\pm 0.2 \\
\mathrm{bc}\end{array}$ & $\underset{\mathrm{bc}}{ \pm 0.1}$ & $\begin{array}{c}0.2 \\
\pm 0.03 \\
\mathrm{c}\end{array}$ & $\underset{\mathrm{bc}}{ \pm 0.02}$ & $\begin{array}{c}0.1 \\
\pm 0.02 \\
\text { bc }\end{array}$ \\
\hline $\mathrm{Cu}$ & $\begin{array}{c}2.5 \\
\pm 0.6 \\
a\end{array}$ & $\begin{array}{c}2.3 \\
\pm 0.5 \\
\mathrm{a}\end{array}$ & $\begin{array}{c}2.2 \\
\pm 0.4 \\
\mathrm{a}\end{array}$ & $\begin{array}{c}2.0 \\
\pm 0.2 \\
a b\end{array}$ & $\begin{array}{c}1.6 \\
\pm 0.3 \\
\text { bc }\end{array}$ & $\begin{array}{c}1.4 \\
\pm 0.2 \\
\text { bc }\end{array}$ & $\begin{array}{c}1.2 \\
\pm 0.6 \\
\mathrm{c}\end{array}$ & $\begin{array}{c}1.7 \\
\pm \underset{c}{ } 1.0\end{array}$ & $\begin{array}{c}1.4 \\
\pm 0.9 \\
c\end{array}$ & $\begin{array}{c}1.1 \\
\pm 0.6 \\
c\end{array}$ & $\begin{array}{c}0.9 \\
\pm 0.5 \\
\mathrm{c}\end{array}$ & $\begin{array}{c}1.0 \\
\pm 0.6 \\
c\end{array}$ \\
\hline Co & $\begin{array}{c}1.4 \\
\pm 1.1 \\
\mathrm{a}\end{array}$ & $\begin{array}{c}1.2 \\
\pm 1.0 \\
a b\end{array}$ & $\begin{array}{c}1.0 \\
\pm 0.9 \\
a b\end{array}$ & $\begin{array}{c}0.9 \\
\pm 0.1 \\
\text { abc }\end{array}$ & $\begin{array}{c}1.2 \\
\pm 1.0 \\
a b\end{array}$ & $\begin{array}{c}1.0 \\
\pm 0.5 \\
a b\end{array}$ & $\begin{array}{c}0.6 \\
\pm 0.1 \\
\mathrm{bc}\end{array}$ & $\begin{array}{c}1.0 \\
\pm 0.2 \\
a b\end{array}$ & $\begin{array}{c}1.0 \\
\pm 0.02 \\
\text { ab }\end{array}$ & $\begin{array}{c}0.9 \\
\pm 0.02 \\
\text { abc }\end{array}$ & $\begin{array}{c}0.5 \\
\pm 0.02 \\
\mathrm{c}\end{array}$ & $\begin{array}{c}0.6 \\
\pm 0.02 \\
\mathrm{bc}\end{array}$ \\
\hline $\mathrm{Cr}$ & $\begin{array}{c}1.6 \\
\pm 1.0 \\
\text { abc }\end{array}$ & $\begin{array}{c}1.4 \\
\pm 0.8 \\
\text { abc }\end{array}$ & $\begin{array}{c}1.2 \\
\pm 0.6 \\
\text { cd }\end{array}$ & $\begin{array}{c}1.0 \\
\pm 0.4 \\
\text { cd }\end{array}$ & $\begin{array}{c}1.4 \\
\pm 0.4 \\
\text { abc }\end{array}$ & $\begin{array}{c}1.1 \\
\pm 0.2 \\
\text { bc }\end{array}$ & $\begin{array}{c}0.9 \\
\pm 0.2 \\
\text { cd }\end{array}$ & $\begin{array}{c}0.6 \\
\pm 0.2 \\
\mathrm{~d}\end{array}$ & $\begin{array}{c}1.0 \\
\pm 0.7 \\
\mathrm{~cd}\end{array}$ & $\begin{array}{c}1.0 \\
\pm 0.6 \\
\text { cd }\end{array}$ & $\begin{array}{c}1.6 \\
\pm 0.5 \\
a b\end{array}$ & $\begin{array}{c}1.8 \\
\pm 0.6 \\
a\end{array}$ \\
\hline
\end{tabular}

Data are presented as means \pm standard deviation $(n=5)$. Sum: summer, Aut: autumn, Win: winter, Spr: spring. Values with different letters in the same row were significantly different $(P<0.05)$.

gaping. Microscopic examination of mantle, gills, gonad and digestive tract of date mussels collected from Abo Qir during summer using light and electron microscope showed that they were infected with Ciliophora. Haemocytes infiltration due to heavy infection as an immune defence of the sample was obvious in gills (Figures 3a). Ciliates were characterized by its oval to spherical shape and large macronucleus under light microscope, and appeared in mussel gills (Figures $3 \mathrm{a}$ and $\mathrm{b}$ ) and gonad (Figure 3c). The ultrastructure of ciliary apparatus of Ciliophoran was clear in
Figures " $3 \mathrm{~d}$ and $\mathrm{e}$ ", where a transverse section of longitudinal bundle of microtubules forming axoneme, which arranged into two arrays of microtubules $9+2$ arrays, was shown. Another abnormal condition appeared in date mussels of polluted area was disseminated sarcomas or haematopoietic neoplastic cells with hypertrophied enlarged cells than normal ones, which appeared in gills (Figure 3a) and digestive tract as two nuclei cell (Figure 3f), or with basophilic dense nuclei (Figure $3 f$ and g) that had high nucleus: cytoplasm ratio. 
Table 3: Heavy metals $(\mathrm{mg} / \mathrm{kg})$ in soft parts of collected Lithophaga lithophaga specimens from the studying area during the four seasons.

\begin{tabular}{|c|c|c|c|c|c|c|c|c|c|c|c|c|}
\hline & \multicolumn{4}{|c|}{ Abo-Qir } & \multicolumn{4}{|c|}{ Alasafra } & \multicolumn{4}{|c|}{ Miami } \\
\hline & Sum & Aut & Win & Spr & $\mathrm{Im}$ & Aut & Win & $\mathrm{or}$ & $\mathrm{m}$ & ut & Win & $\mathrm{pr}$ \\
\hline $\mathrm{Fe}$ & $\begin{array}{c}5.4 \\
\pm 3.4 \\
\mathrm{a}\end{array}$ & $\begin{array}{c}5.2 \\
\pm 3.2 \\
\mathrm{ab}\end{array}$ & \pm 3.0 & $\begin{array}{r}5.3 \\
\pm 3 . \\
\text { ab }\end{array}$ & 9 & $\begin{array}{c} \pm 0.6 \\
\mathrm{abc}\end{array}$ & \pm 0 & & & ${ }_{\mathrm{cd}}^{ \pm 1.0}$ & \pm 0.5 & $\begin{array}{c}3.7 \\
\pm 0.8 \\
\mathrm{~cd}\end{array}$ \\
\hline $\mathrm{Ni}$ & $\begin{array}{c}0.9 \\
\pm 0.1 \\
\mathrm{a}\end{array}$ & $\begin{array}{c}0.7 \\
\pm 0.3 \\
\mathrm{ab}\end{array}$ & $\begin{array}{c}0.6 \\
\pm 0.5 \\
\text { bc }\end{array}$ & $\begin{array}{r}0.4 \\
\pm 0.3 \\
\mathrm{c}\end{array}$ & $\underset{\mathrm{bc}}{ \pm 0.4}$ & $\begin{array}{c}0.4 \\
\pm 0.2 \\
\mathrm{c}\end{array}$ & $\begin{array}{r}0 \\
\pm 0 \\
\mathrm{c}\end{array}$ & $\begin{array}{c}0.4 \\
\pm 0.2 \\
\mathrm{c}\end{array}$ & $\begin{array}{c}1.0 \\
\pm 0.3 \\
a\end{array}$ & $\begin{array}{c}0.8 \\
\pm 0.1 \\
\mathrm{a}\end{array}$ & $\begin{array}{c}0.7 \\
\pm 0.1 \\
\mathrm{ab}\end{array}$ & $\begin{array}{c}0.8 \\
\pm 0.3 \\
\mathrm{a}\end{array}$ \\
\hline $\mathrm{Pb}$ & $\begin{array}{c}2.1 \\
\pm 1.5 \\
a b\end{array}$ & $\begin{array}{c}1.9 \\
\pm 1.2 \\
a b\end{array}$ & $\begin{array}{c}2.0 \\
\pm 1.4 \\
\mathrm{a}\end{array}$ & $\begin{array}{c}1.7 \\
\pm 1.0 \\
\mathrm{~b}\end{array}$ & $\begin{array}{c}0.9 \\
\pm 0.6 \\
\mathrm{c}\end{array}$ & $\begin{array}{c}0.9 \\
\pm 0.6 \\
\text { cd }\end{array}$ & $\begin{array}{c}0.5 \\
\pm 0.04 \\
\mathrm{e}\end{array}$ & $\begin{array}{r}0.5 \\
\pm 0.0 \\
\mathrm{e}\end{array}$ & $\begin{array}{c}0.8 \\
\pm 0.3 \\
\text { cde }\end{array}$ & $\begin{array}{c}0.6 \\
\pm 0.1 \\
\text { de }\end{array}$ & $\begin{array}{c}0.6 \\
\pm 0.2 \\
\text { cde }\end{array}$ & $\begin{array}{c}0.6 \\
\pm 0.3 \\
\text { cde }\end{array}$ \\
\hline $\mathrm{Zn}$ & $\begin{array}{c}5.3 \\
\pm 2.1 \\
\mathrm{a}\end{array}$ & $\begin{array}{c}5.0 \\
\pm 2.0 \\
\mathrm{a}\end{array}$ & $\begin{array}{c}4.9 \\
\pm 1.8 \\
\mathrm{a}\end{array}$ & $\begin{array}{c}4.9 \\
\pm 1.8 \\
\mathrm{a}\end{array}$ & $\begin{array}{c}5.0 \\
\pm 0.1 \\
\mathrm{a}\end{array}$ & $\begin{array}{c}4.5 \\
\pm 0.1 \\
\mathrm{a}\end{array}$ & $\begin{array}{c}4.4 \\
\pm 0.1 \\
\mathrm{a}\end{array}$ & $\begin{array}{c}4.5 \\
\pm 0.1 \\
\mathrm{a}\end{array}$ & $\begin{array}{c}4.9 \\
\pm 1.0\end{array}$ & $\begin{array}{c}4.5 \\
\pm 1.8 \\
\mathrm{a}\end{array}$ & $\begin{array}{c}4.2 \\
\pm 1.6 \\
\mathrm{a}\end{array}$ & $\begin{array}{c}4.5 \\
\pm 1.8 \\
\mathrm{a}\end{array}$ \\
\hline $\mathrm{Cd}$ & $\begin{array}{c}2.4 \\
\pm 0.1 \\
\mathrm{a}\end{array}$ & $\begin{array}{c}0.9 \\
\pm 0.01 \\
\mathrm{~cd}\end{array}$ & $\begin{array}{c}0.8 \\
\pm 0.01 \\
\mathrm{~d}\end{array}$ & $\begin{array}{c}1.0 \\
\pm \underset{\mathrm{cd}}{0.02}\end{array}$ & $\pm{ }_{\mathrm{cd}} 1.1$ & $\begin{array}{c}2.2 \\
\pm 1.2 \\
\mathrm{a}\end{array}$ & $\underset{a b}{ \pm 0.8}$ & $\underset{\mathrm{ab}}{ \pm 1.0}$ & $\underset{\mathrm{bc}}{ \pm 0.5}$ & $\underset{\mathrm{cd}}{ \pm 0.3}$ & \pm 0.2 & $\begin{array}{c}1.0 \\
\pm 0.3 \\
\mathrm{~cd}\end{array}$ \\
\hline $\mathrm{Cu}$ & $\begin{array}{c}4.2 \\
\pm 0.9 \\
\mathrm{a}\end{array}$ & \pm 0.6 & \pm 0.6 & \pm 0.8 & $\begin{array}{c}2.9 \\
\pm 1.0 \\
\mathrm{bc}\end{array}$ & $\begin{array}{c}2.6 \\
\pm 0.9 \\
\text { cd }\end{array}$ & $\pm_{\mathrm{cd}} 0.5$ & $\pm_{\mathrm{cd}} 0.7$ & $\pm_{\mathrm{cd}} 0.3$ & $\pm_{\mathrm{cd}}$ & $\pm_{\mathrm{cd}} 0.3$ & $\begin{array}{c}2.0 \\
\pm 0.02 \\
\mathrm{~d}\end{array}$ \\
\hline Co & $\begin{array}{c}0.9 \\
\pm 0.5 \\
\mathrm{a}\end{array}$ & $\begin{array}{c}0.8 \\
\pm 0.4 \\
\mathrm{a}\end{array}$ & $\begin{array}{c}0.5 \\
\pm 0.02 \\
\mathrm{a}\end{array}$ & $\begin{array}{c}0.5 \\
\pm 0.1 \\
\mathrm{ab}\end{array}$ & $\begin{array}{c}0.8 \\
\pm 0.3 \\
a\end{array}$ & $\begin{array}{c}0.7 \\
\pm 0.1 \\
a b\end{array}$ & $\begin{array}{c}0.6 \\
\pm 0.04 \\
a b\end{array}$ & $\begin{array}{c}0.6 \\
\pm 0.1 \\
a b\end{array}$ & $\begin{array}{c}0.6 \\
\pm 0.02 \\
\mathrm{~b}\end{array}$ & $\begin{array}{c}0.5 \\
\pm 0.02 \\
\mathrm{~b}\end{array}$ & \pm 0.1 & $\begin{array}{c}0.5 \\
\pm 0.01 \\
\mathrm{~b}\end{array}$ \\
\hline $\mathrm{Cr}$ & $\begin{array}{c}1.3 \\
\pm 1.0 \\
\mathrm{a}\end{array}$ & $\begin{array}{c}0.9 \\
\pm 0.1 \\
\text { bcd }\end{array}$ & $\begin{array}{c}0.7 \\
\pm 0.6 \\
\text { cd }\end{array}$ & $\begin{array}{c}0.9 \\
\pm 0.1 \\
\text { bcd }\end{array}$ & $\begin{array}{c}0.9 \\
\pm 0.5 \\
\text { bcd }\end{array}$ & $\begin{array}{c}0.8 \\
\pm 0.4 \\
\text { bcd }\end{array}$ & $\begin{array}{c}0.6 \\
\pm 0.3 \\
d\end{array}$ & $\begin{array}{c}0.6 \\
\pm 0.2 \\
\mathrm{~d}\end{array}$ & $\begin{array}{c}0.7 \\
\pm 0.3 \\
\mathrm{ab}\end{array}$ & $\begin{array}{c}0.6 \\
\pm 0.2 \\
\text { bcd }\end{array}$ & $\begin{array}{c}0.5 \\
\pm 0.1 \\
\text { bcd }\end{array}$ & $\begin{array}{c}0.6 \\
\pm 0.1 \\
\text { abc }\end{array}$ \\
\hline
\end{tabular}

Data are presented as means \pm standard deviation $(n=5)$. Sum: summer, Aut: autumn, Win: winter, Spr: spring. Values with different letters in the same row were significantly different $(P<0.05)$.

\section{DISCUSSION}

Marine pollution has increased recently in the worldwide. It could affect the ecosystem and cause long term, negative effects on the surrounding marine environment $^{[12]}$. Pollution with heavy metals in the aquatic environment increased recently under the effect of sewage and industrial effluent ${ }^{[13]}$. Mussels are recognized as a pollutant bio-indicator because they accumulate high levels of pollutant in their tissues from the surrounding marine environment ${ }^{[3]}$. The bioaccumulation of heavy metals in mussels' tissues depends on their concentration in the environment, in addition to biological and environmental factors ${ }^{[2]}$. The deposition of contaminants into a marine environment was possibly due to the transport of contaminants from upstream coastal industrial activities ${ }^{[14]}$. The present study showed that $\mathrm{Pb}, \mathrm{Cd}$, and $\mathrm{Cu}$ increased significantly in Abo Qir during summer compared with the other coastal sites, Miami and Alasafra. Radwan et al. ${ }^{[15]}$ also found that Abo Qir seawater mostly polluted with $\mathrm{Cu}$ and $\mathrm{Cd}$ compared with the seawater collected from Miami and El Mex. 
Table 4: The relation between heavy metals, site of collection, and season in both seawater samples and soft parts of Lithophaga lithophaga samples.

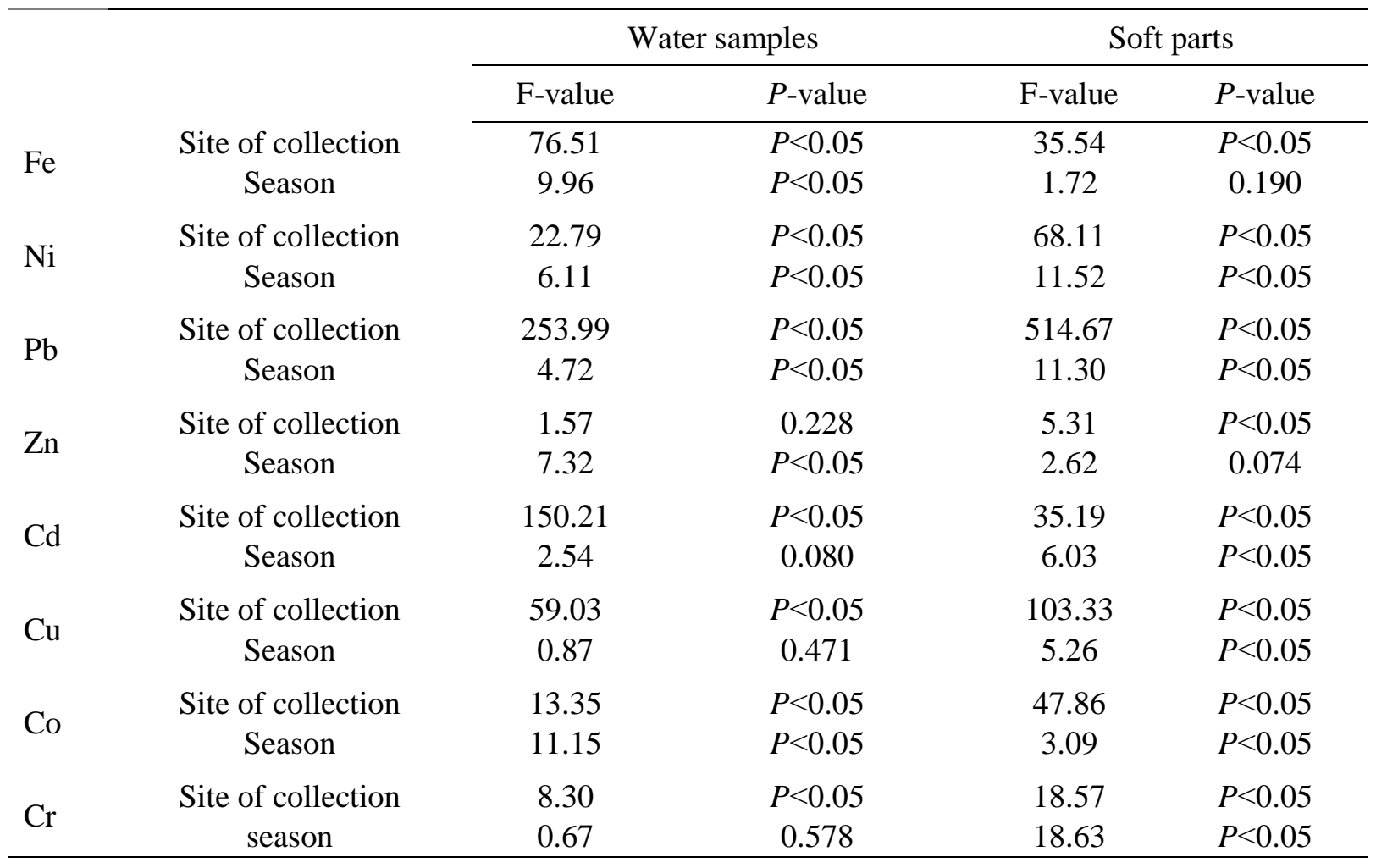
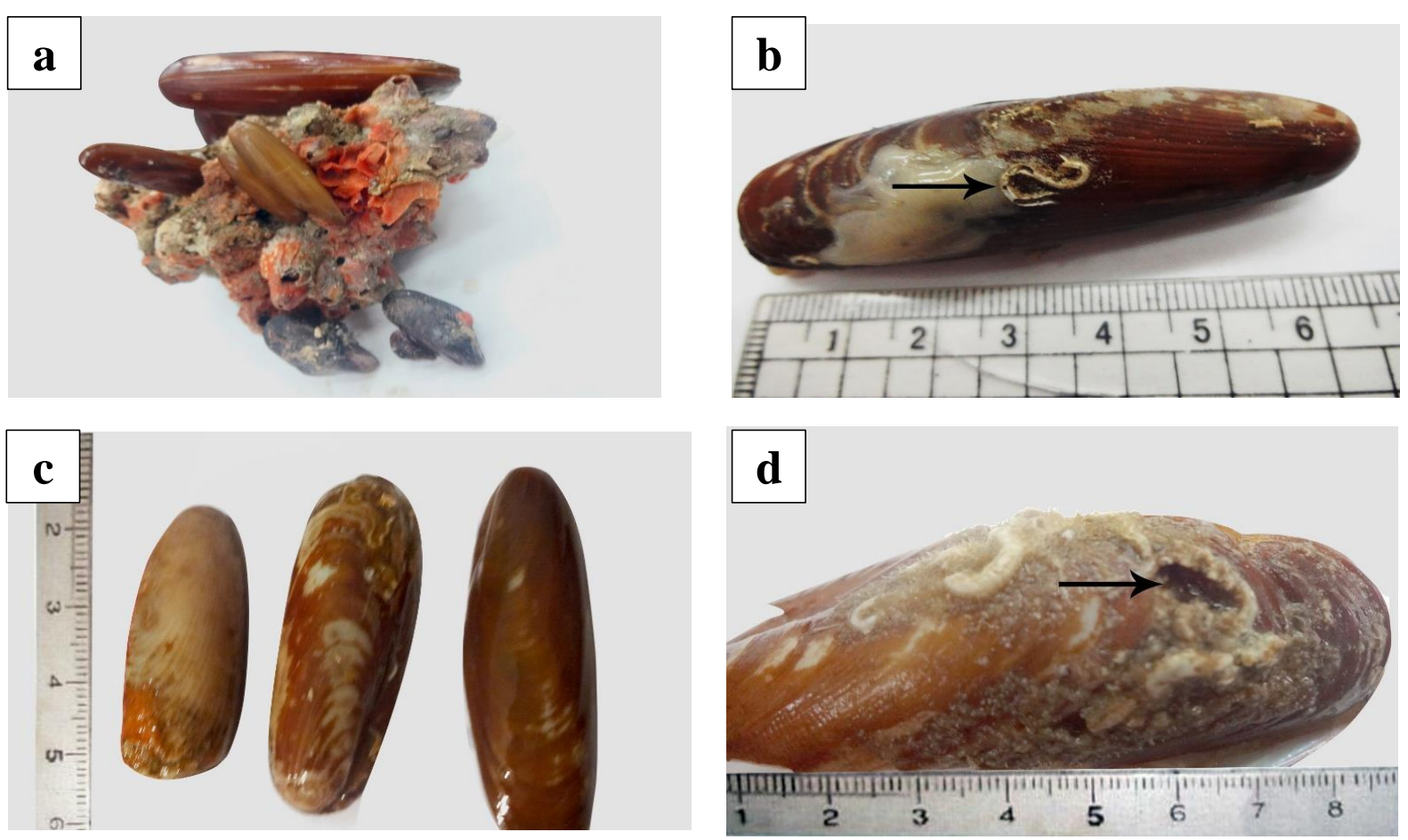

Figure 2: Photomicrograph showing signs of weakness of Lithophaga lithophaga collected from Abo Qir. Their outer shell surfaces showed (a and b) fouling (black arrow indicates fouling of hydroid tube), (c) degrees of discolouration, (d) deformation and fragility (black arrow indicates holes and fragility). 

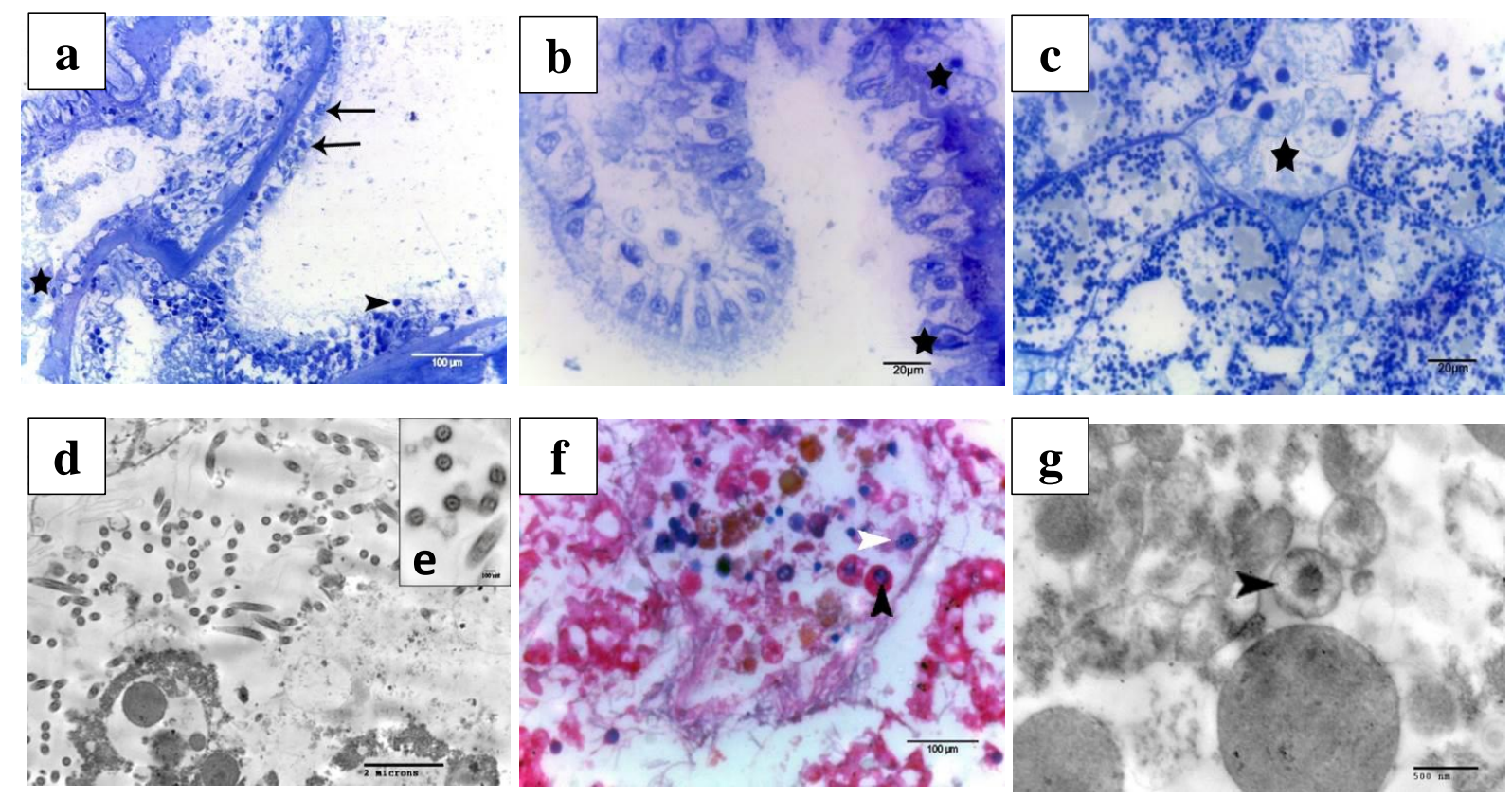

Figure 3: Histological and ultrastructure of soft parts of Lithophaga lithophaga collected from Abo Qir area showing: (a) haemocytes infiltration (black arrows), neoplastic cells with pleomorphic nuclei (black arrow head) and ciliates (star) in gills of date mussel, bar $=100 \mu \mathrm{m},(\mathbf{b})$ ciliates embedded in gills, bar $=20 \mu \mathrm{m},(\mathbf{c})$ ciliates in gonad, bar $=20 \mu \mathrm{m}$, (d) ultrastructure of ciliary apparatus of ciliates in the digestive tract of mussel, bar $=100$ $\mathrm{nm}$, with axoneme arranged in two arrays of microtubules in transverse section (e), (f) neoplastic cells in digestive tract of date mussel showing cell with 2 nuclei (white arrow head) and basophilic nucleus (black arrow head), bar $=100 \mu \mathrm{m},(\mathbf{g})$ transmission electron microscopy showing cell with the basophilic nuclei (black arrow head), bar = $500 \mathrm{~nm}$.

On the other hand, MacFarlane et al. ${ }^{[16]}$ stated that Egyptian coastal area of Mediterranean Sea might be considered relatively unpolluted with heavy metals. The results of current study were evidenced by the fact that, Abo-Qir station in Alexandria was polluted by the disposal of effluents of both sewage and industrial activities. Ramadan et $a l^{[17]}$ found that Abo Qir station was affected by three discharges as Boughaz El-Maadia opening, Tapia pumping station and the opening of Rashid Nile branch. The elevation of $\mathrm{Cu}$ level in Abo Qir seawater shown in the current study might be due to leaching of $\mathrm{Cu}$ from ships antifouling paints. Furthermore, $\mathrm{Cd}$ showed high concentration in the Abo Qir seawater in the present work during the four seasons. It was also found that leakage from ships painting, in addition to agriculture, sewage, and industrial waste effluents led to high concentration of $\mathrm{Pb}^{[18]}$.

As a consequence of water pollution in the studying coastal areas of Alexandria in the current study, soft parts of date mussels accumulate heavy metals in their tissue. Giustui et al. ${ }^{[19]}$ confirmed that marine organisms' tissues in polluted area could accumulate heavy metals. Bivalves could serve as pollution bio-indicators for metals in Egyptian water ${ }^{[20]}$. Fe is more concentrated in date mussels' tissues followed by $\mathrm{Zn}>\mathrm{Cu}>\mathrm{Cd}>\mathrm{Pb}>\mathrm{Cr}>\mathrm{Co}$ and $\mathrm{Ni}$ in the current study. Abdel Gawad ${ }^{[21]}$ also detected the accumulation of $\mathrm{Fe}$ in soft tissue of Lanistas carinatus that dwell in rock surfaces collected from Manzala Lake, Egypt. Freshwater Mollusca could accumulate $\mathrm{Fe}, \mathrm{Cd}, \mathrm{Pb}, \mathrm{Mn}, \mathrm{Cu}$, and $\mathrm{Zn}$ in their tissues ${ }^{[22]}$. The ability of metal 
accumulation in soft parts differs from one species to another according to rate of adsorption and digestion, or according to the pollution level, metabolic rate, and age of the snail ${ }^{[22,23]}$. On the other hand, pollution did not significantly affect protein, carbohydrates, moisture, fibre, and ash contents, while lipid content somehow decreased, in the date mussels collected from Abo Qir (data not shown). Other study reported a decrease in lipid contents of stressed aquatic organisms in polluted area due to consuming of energy during eliminating the toxins from their bodies ${ }^{[24,25]}$. Heavy metals accumulation was affected by temperature, salinity, diet, and variations in marine organisms ${ }^{[26]}$. Water quality depends on the adequate dissolved oxygen; its ratio below $5 \mathrm{mg} / \mathrm{L}$ could cause stress to aquatic life ${ }^{[27]}$. The main causes of decreasing the dissolved oxygen concentration in seawater are the elevation in seawater temperature, algal bloom, animal and human wastes ${ }^{[27]}$. As a consequence of pollution in Abo Qir during summer, the seawater in this area showed the lowest value of dissolved oxygen $(4.0 \pm 0.2 \mathrm{mg} / \mathrm{L})$.

Pollutant and metals have a great effect on the immune system of aquatic organisms and could increase susceptibility to infection ${ }^{[28]}$. Therefore, the bivalved Mollusca in polluted areas were vulnerable to infection with a lot of pathogens. In the current study, there were no records for infection with white spot syndrome, herpeslike, and irido-like viruses in date mussels collected from Abo Qir (data not shown). In contrary, viral pollution was recorded in different species of wild and farmed bivalve molluscs in Alaska ${ }^{[29]}$. According to World Organization for Animal Health, Mollusca could be infected with protozoan parasites of genera Perkinsus, Bonamia, Martellia, and Haplosporidium that induced mortality and caused enormous losses in harvested molluscan species population ${ }^{[11]}$. Prevalence could increase due to a lot of factors as high temperature in warm season, high salinity, and pollution ${ }^{[30]}$. In the current study, ciliates were easily defined in gills and gonads of Abo Qir date mussels by TEM, as pear shaped, with large macronucleus and its cilia showed axonemes arrangement $(9+2)$. In the northeast coast of America, gill ciliates were also found in bivalvia collected from the heavily polluted sites and the body burden was correlated with the degree of pollutions ${ }^{[31]}$. Gill ciliates in mussels had a negative correlation with $\mathrm{Ni}$ contamination, but were positively correlated with $\mathrm{Cd}$ and selenium contamination ${ }^{[32]}$. As the progress of sarcoma (neoplasia of mesoderm-derived tissues), the haemocytes cells were replaced by the neoplastic cells with pleomorphic or basophilic nucleus, which unable to create the function of normal cells as phagocytosis and defence function, therefore the aquatic organisms become more susceptible to disease ${ }^{[33,34]}$. In the current study date mussels samples form Abo Qir showed neoplastic cells in digestive tract. The same finding was obtained by another study on Mediterranean mussels "Mytilus galloprovincialis" collected from Slovene Adriatic $\mathrm{Sea}^{[35]}$. Necrosis and multifocal atrophy of digestive tubules were noticed in mussels with a diffuse neoplasia, whereas severe haemocytic infiltration of connective tissue was seen in mussels with single neoplastic cells ${ }^{[35]}$. Possible cause of the haemocytic neoplasia is a viral infection, but other factors such as marine pollution and biotoxins may be involved ${ }^{[35]}$. Noticeably, no viral infection was recorded in the present study in the date mussels collected from the most polluted area "Abo Qir" in summer that showed neoplastic cells in digestive tract; therefore, the heavy metals pollution may be the main cause for resulted disseminated sarcoma.

In conclusion, the most polluted seawater area with the heavy metals was the Abo Qir in summer in the current study. The seawater of this area was characterized by a decrease in the amount of dissolve oxygen. In addition, Lithophaga lithophaga samples collected form Abo Qir showed accumulation of heavy metals (especially $\mathrm{Pb}$, $\mathrm{Cd}$, and $\mathrm{Cu}$ ) and neoplastic cells in their 
soft parts, a decrease in immunity, and an increase in the susceptibility to ciliates infection

\section{ACKNOWLEDGMENTS}

This research received no specific grant from any funding agency in the public, commercial or not-for-profit sectors.

\section{CONFLICT OF INTEREST}

The authors have no potential financial conflict of interest.

\section{AUTHORS' CONTRIBUTIONS}

WMS and MHM proposed the research idea, designed the experiments. WMS and AZG performed the practical work, analysed the data, reviewed the literatures and data collection, as well as drafted the manuscript.

\section{REFERENCES}

[1] El-Menif, N. T.; Kefi, F. J.; Ramdani, M. et al. (2007). Habitat and associated fauna of Lithophaga lithophaga (Linné 1758) in the Bay of Bizerta (Tunisia). Journal of Shellfish Research, 26(2): 569-574.

[2] Ali, H.; Khan, E. and Ilahi, I. (2019). Environmental chemistry and ecotoxicology of hazardous heavy metals: environmental persistence, toxicity, and bioaccumulation. Journal of Chemistry, 2019: 6730305 (DOI: $10.1155 / 2019 / 6730305)$.

[3] Azizi, G.; Akodad, M.; Baghour, M. et al. (2018). The use of Mytilus spp. mussels as bioindicator of heavy metal pollution in the coastal environment. A review. J Mater Environ Sci, 9(4): 1170-1181.

[4] Wong, S.; Fournier, M.; Coderre, D. et al. (1992). Environmental immunotoxicology. In: Animals Biomarkers as Pollution Indicators (Peakall, D., ed), pp. 167-189. Chapman and Hall, London, UK.

[5] Morley, N. J. (2009). Environmental risk and toxicology of human and veterinary waste pharmaceutical exposure to wild aquatic hostparasite relationships. Environ Toxicol Pharmacol, 27(2): 161-175.

[6] Fernández Robledo, J. A.; Vasta, G. R. and Record, N. R. (2014). Protozoan parasites of bivalve molluscs: literature follows culture. PLOS ONE, 9(6): e100872 (DOI: 10.1371/journal.pone. 0100872).

[7] Namroodi, S.; Milanlou, D. and Rezaei, H. (2017). Heavy metal bioaccumulation and its potential relation with incidence of canine parvovirus infection in golden jackals, North Iran. Iran J Vet Med, 11(2): 113-124.

[8] Barber, B. J. (2004). Neoplastic diseases of commercially important marine bivalves Aquat Living Resour, 17: 449-466.

[9] Grasshof, K. (1976). Methods of Seawater Analysis. Verlag Chemie, Weinhein, Germany.

[10] Bernhard, M. (1976). Manual of Methods in Aquatic Environment Research. Part 3: Sampling and Analysis of Biological Material. Food and Agriculture Organization of the United Nation, Rome, Italy.

[11] OIE (2012). Manual of Diagnostic Tests for Aquatic Animals. World Organisation for Animal Health, Paris, France.

[12] Catsiki, V. A. and Florou, H. (2006). Study on the behaviour of the heavy metals $\mathrm{Cu}, \mathrm{Cr}, \mathrm{Ni}, \mathrm{Zn}, \mathrm{Fe}$, $\mathrm{Mn}$ and 137Cs in an estuarine ecosystem using Mytilus galloprovincialis as a bioindicator species: the case of Thermaikos gulf, Greece. Journal of Environmental Radioactivity, 86: 3144.

[13] Huanxin, W.; Lejun, Z. and Presley, B. J. (2000). Bioaccumulation of heavy metals in oyster (Crassostrea virginica) tissue and shell. Environ Geol, 39(11): 1216-1226.

[14] Madkour, H. A. (2005). Distribution and relationships of heavy metals in 
the gaint clam (Tridacna maxima) and associated sediments from different sites in the Egyptian Red Sea coast. Egyptian Journal of Aquatic Research, 31(2): 45-59.

[15] Radwan, E. H.; Hamed, S. S. and Saad, G. A. (2014). Temporal and spatial effects on some physiological parameters of the bivalve Lithophaga lithophaga (Linnaeus, 1758) from coastal regions of Alexandria, Egypt. Open Journal of Ecology, 4: 732-743.

[16] MacFarlane, G. R.; Markich, S. J.; Linz, K. et al. (2005).The Akoya pearl oyster shell as an archival monitor of lead exposure. Environ Pollut, 143: 166-173.

[17] Ramadan, SH. E.; Kheirallah, A. M. and Abdel-Salam, KH. M. (2006). Factors controlling marine fouling in some Alexandria Harbours, Egypt. Medit Mar Sci, 7(2): 31-54.

[18] Zyadah, M.; Abdel-Baky, T.; Ibrahim, M. et al. (2002). Assessment of some heavy metal pollutants in water and sediment at Damietta Mediterranean coast, Egypt. J Union Arab Biol, 17: 277-292.

[19] Giusti, L.; Williamson, A. C. and Mistry, A. (1999). Biologically available trace metals in Mytilus edulis from the coast of northeast England. Environ Int, 25(8): 969-981.

[20] Khairy, M. A.; Kotb, M.; Schimdt, C. et al. (2011). Trace elements in sediments and mussels - spatial distribution, chemical partitioning, and risk assessment. Clean - Soil, Air, Water, 39(3): 301-312.

[21] Abd El Gawad, S. S. (2018). Concentrations of heavy metals in water, sediment and mollusk gastropod, Lanistes carinatus from Lake Manzala, Egypt. Egyptian Journal of Aquatic Research, 44(2): 77-82.

[22] Cravo, A.; Bebianno, M. J. and Foster, P. (2004). Partitioning of trace metals between soft tissues and shells of Patella aspera. Environ Int, 30: 87-98.
[23] Ndome, C. B.; Ekaluo, U. B. and Asuquo, F. E. (2010). Comparative bioaccumulation of heavy metals $(\mathrm{Fe}$, $\mathrm{Mn}, \mathrm{Zn}, \mathrm{Cu}, \mathrm{Cd}$, and $\mathrm{Cr}$ ) by some edible aquatic molluscs from the Atlantic coastline of South Eastern Nigeria. World J Fish \& Marine Sci, 2(4): 317-321.

[24] Salman, J. M. and Nasar, A. J. (2013). Total lipids and total protein in two mollusca species as environmental biomarkers of pollution in Euphrates River, Iraq. Int J Curr Microbiol Appl Sci, 2(10): 207-214.

[25] De Coen, W. M. and Janssen, C. R. (2003). The missing biomarker link: relationships between effects on the cellular energy allocation biomarker of toxicant-stressed Daphnia magna and corresponding population characteristics. Environ Toxicol Chem, 22(7): 1632-1641.

[26] El-Moselhy, K. M. and Yassien, M. H. (2005). Accumulation patterns on heavy metals in venus clams, Paphia undulate (Born, 1780) and Gafrarium pectinatum (Linnaeus, 1758), from Lake Timsah, Suez Canal, Egypt. Egyptian Journal of Aquatic Research, 31: 13-28.

[27] Clean Water Team (2004). Dissolved oxygen fact sheet, FS-3.1.1.0 (DO). In: The Clean Water Team Guidance Compendium for Watershed Monitoring and Assessment, Version 2.0. Division of Water Quality, California State Water Resources Control Board (SWRCB), Sacramento, CA.

[28] Yokoyama, A. and Park, H. D. (2003). Depuration kinetics and persistence of the cyanobacterial toxin microcystin-LR in the freshwater bivalve Unio douglasiae. Environ Toxicol, 18: 61-67.

[29] Meyers, T. R.; Burton, T.; Evans, W. et al. (2009). Detection of viruses and virus-like particles in four species of wild and farmed bivalve 
molluscs in Alaska USA, from 19872009. Dis Aquat Organ, 88: 1-12.

[30] Kim, Y. and Powell, E. N. (2007). Distribution of parasites and pathologies in sentinel bivalves: NOAA status and trends "Mussel watch" program. Journal of Shellfish Research, 26(4): 1115-1151

[31] Zannella, C.; Mosca, F.; Mariani, F. et al. (2017). Microbial diseases of bivalve mollusks: infections, immunology and antimicrobial defense, Mar Drugs, 15(6): E182 (DOI: 10.3390/md15060182).

[32] Kim, Y.; Powell, E. N.; Wade, T. L. et al. (1998). Parasites of sentinel bivalves in the NOAA status and trends program: distribution and relationship to contaminant body burden. Marine Pollution Bulletin, 37 (1-2): 45-55.

[33] Metzger, M. J.; Reinisch, C.; Sherry, J. et al. (2015). Horizontal transmission of clonal cancer cells causes leukemia in soft-shell clams. Cell, 161(2): 255263.

[34] Metzger, M. J.; Villalba, A.; Carballal, M. J. et al. (2016). Widespread transmission of independent cancer lineages within multiple bivalve species. Nature, 534: 705-709.

[35] Gombač, M.; Sitar, R.; Pogačnik, M. et al. (2013). Haemocytic neoplasia in Mediterranean mussels (Mytilus galloprovincialis) in the Slovene Adriatic Sea. Marine Freshwater Behaviour Physiology, 46(2): 135-143.

\section{How to cite this article:}

Salama, W. M.; Mona, M. H. and Ghoneium, A. Z. (2020). Health status of date mussel "Lithophaga Lithophaga, Family: Mytildae" in Alexanderia, Egypt: potential effects of the heavy metal pollution. Egyptian Journal of Zoology, 73: 26-38 (DOI: 10.12816/ejz.2020.25041.1022). 


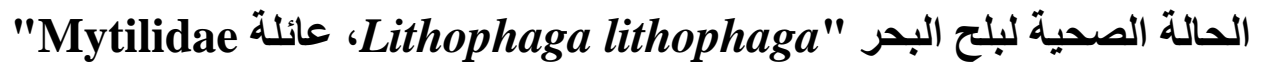

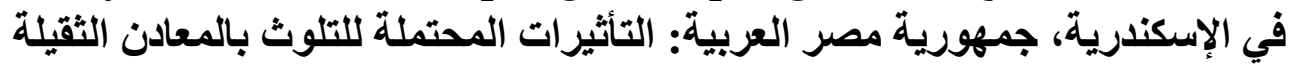

\section{وسام محمد سلامه'1، محمد حسن منا 1، أمل ذكي غنيم²}

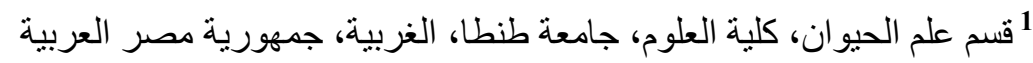

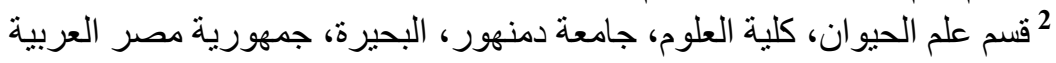

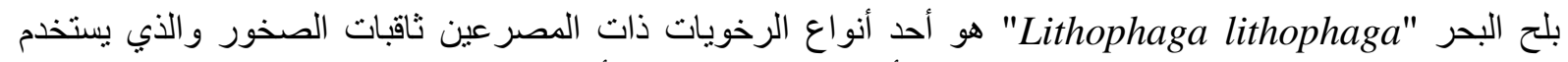

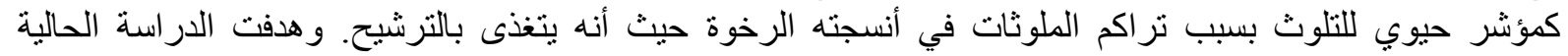

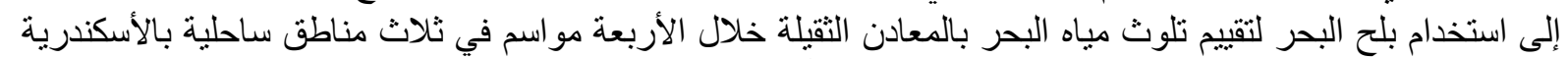

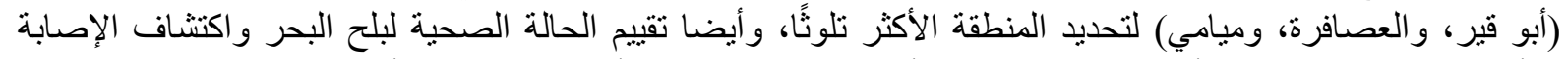

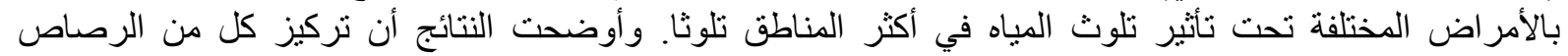

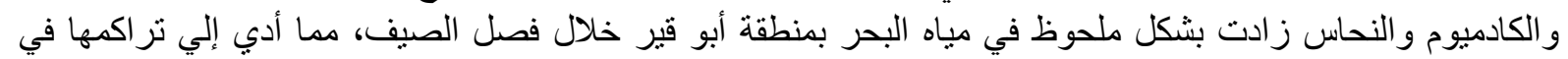

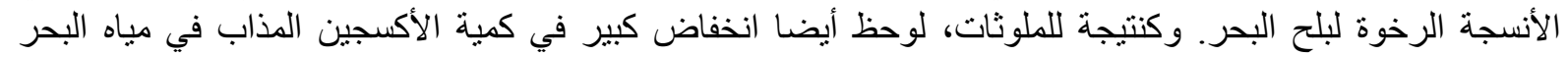

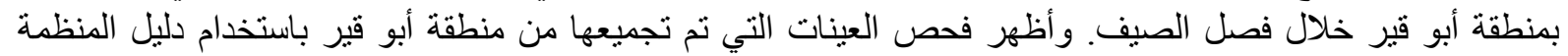

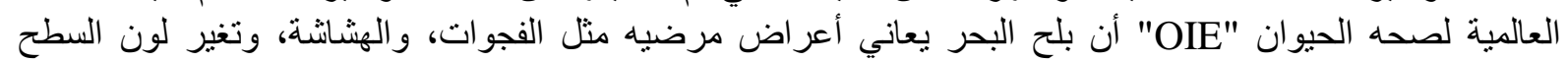

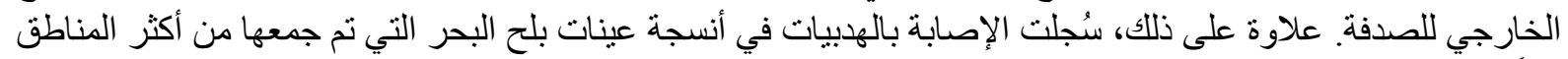

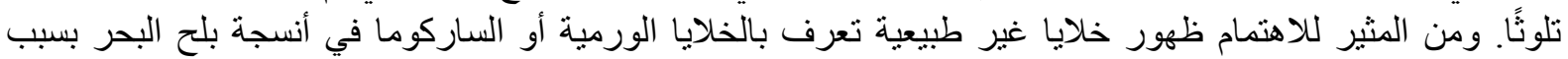

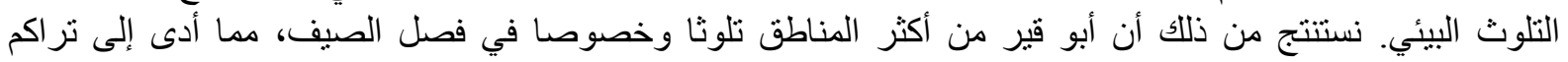

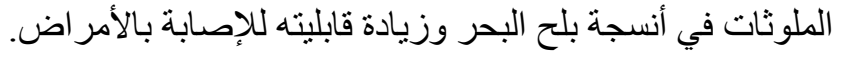

